\title{
Characteristics of Waveguides for Long-Distance Transmission ${ }^{1}$
}

\author{
A. E. Karbowiak and L. Solymar
}

(May 23, 1960)

\begin{abstract}
Discussion of a waveguide communication system is given and the system is compared with existing communication media. The principal properties of waveguide - the medium of communication - are discussed in some detail. The particular significance of helical and coated waveguides is pointed out and the design formulas included. The phenomenon of mode conversion-reconversion, which is peculiar to a waveguide communication system, is discussed in general and the basic theory as applicable to design is also discussed. Design features of components such as bends, transducers, tapers, etc., are analyzed. The effect of waveguide discontinuities is analyzed in some detail and various aspects of signal distortion are also considered.
\end{abstract}

\section{Introduction}

Man, in his constant efforts towards progress, finds it necessary to establish better, quicker, and more numerous communication systems. It is even said that complexity of communication systems grows in proportion to the development of civilization. As the conventional means of communication become gradually used to capacity and the traffic continues to increase, one, naturally, turns towards more exotic means of communication. Waveguide is a possible winner $[1,2,3]^{2}$ and in this connection special acknowledgment should be made of the very important pioneer and continuing work of the Bell Laboratories in this field. Although the final assessment can only be made against the background of economy, a detailed performance assessment must be the first step. For a number of reasons, as we shall see subsequently, such a waveguide is essentially a circular tube about 1 to $3 \mathrm{in}$. in diameter and the waves involved are in the millimetric band. Such a pipe-the medium of communications - would be laid in the ground or at the bottom of oceans for hundreds and thousands of miles.

In order to bring out any advantages that a waveguide communication system may have over the existing systems, the principal features are shown tabulated in table 1 . From this it is evident that the main advantage of a waveguide communication system is its large communication capacity. Provided that very short millimetric waves are used then, in principle, several hundreds of television channels, or the equivalent in speech channels, can be accommodated in a single pipe. The waveguide is also characterized by small attenuation, and in contrast to microwave links it is a screened system. Whether such enormous communication capacities will ever be required is another matter; but, if present statistics are anything to go by, then these capacities will be required in less than 20 years time,

1 Contribution from Standard Telecommunication Laboratories, Ltd., London Road, Harlow, Essex, England.

${ }^{2}$ Figures in brackets indicate the literature references at the end of this papec if not for telephone communication then for purposes such as data transmission, color and cinema television as well as for purposes not yet conceived.

A waveguide communication system is essentially simple (fig. 1). The video channels are grouped together (say by frequency division) and passed to a coder $(C)$ where, for reasons to be discussed, it is coded in PCM (pulse code modulation). The coded signal is then made to activate an on-off modulator $(M)$ operating on one of the many carriers in the whole spectrum carried by the waveguide. The carriers are combined through a suitable channel insertion filter and passed through a transducer $(T)$ on to the waveguide. At convenient intervals along the waveguide (say every 10 miles) regenerative repeaters are inserted to amplify and restore the quality of the signal.

For effective operation the terminal equipment (modulation/demodulation and coding/decoding) is designed to handle millimicrosecond pulses. This in itself is a complication but it is a necessary one to achieve PCM coding of the wide video band and

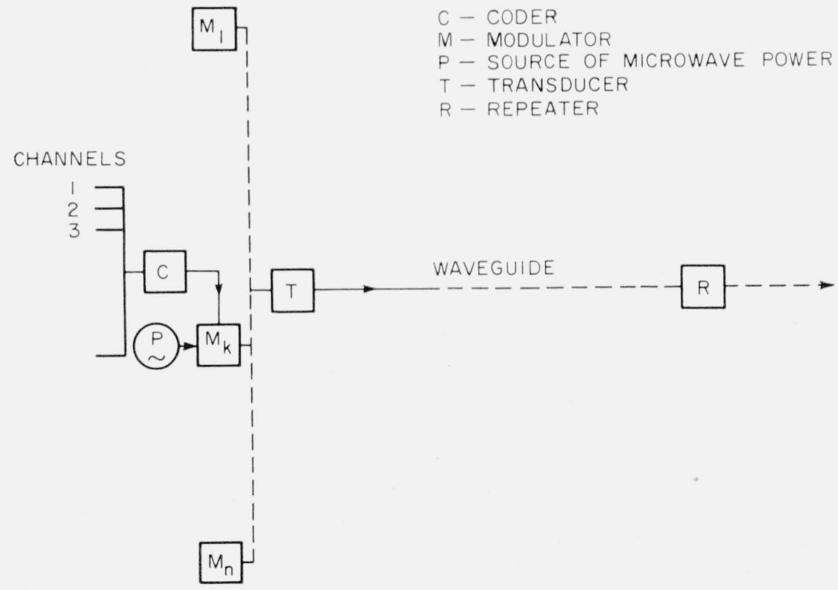

Figure 1. A possible communication system using waveguide. 
TABLE 1. Comparison of communication media

Note: One super channel=4 Me/s.

\begin{tabular}{|c|c|c|c|c|}
\hline \multicolumn{2}{|l|}{ Type } & Operating frequency & Channel capacity & $\frac{\text { Repeater spacing }}{\text { attenuation }}$ \\
\hline \multirow[b]{2}{*}{$\begin{array}{l}\text { Open } \\
\text { wire }\end{array}$} & One pair & $\begin{array}{l}36 \text { to } 84 \mathrm{kc} / \mathrm{s} \mathrm{(go)} \\
92 \text { to } 140 \mathrm{kc} / \mathrm{s} \text { (return) }\end{array}$ & $12+3+(1)=15+(1)$ & \multirow{2}{*}{$\begin{array}{l}60 \text { to } 70 \text { miles } \\
(0.4 \mathrm{db} / \mathrm{mile})\end{array}$} \\
\hline & $\begin{array}{l}\text { Maximum } \\
16 \text { pairs }\end{array}$ & As above & $240+(16)$ & \\
\hline \multirow[t]{2}{*}{$\begin{array}{l}\text { Balance } \\
\text { pair }\end{array}$} & One pair & 12 to $252 \mathrm{kc} / \mathrm{s}$ & 60 & \multirow{2}{*}{$\begin{array}{l}12 \text { to } 30 \text { miles } \\
(4 \mathrm{db} / \mathrm{mile})\end{array}$} \\
\hline & $\begin{array}{l}\text { Maximum } \\
24 \text { pairs }\end{array}$ & As above & 1440 & \\
\hline \multirow{2}{*}{$\begin{array}{l}\text { Coax } \\
\text { G. B. }\end{array}$} & & Up to $4 \mathrm{Mc} / \mathrm{s}$ & $\begin{array}{l}960 \\
\text { (One super channel) }\end{array}$ & 6 miles \\
\hline & & $\mathrm{Up}$ to $12 \mathrm{Mc} / \mathrm{s}$ & 3 super channels & 3 miles \\
\hline \multirow{2}{*}{$\begin{array}{l}\text { Coas } \\
\text { U.S. }\end{array}$} & & Up to about $3 \mathrm{Mc} / \mathrm{s}$ & 720 & 8 miles \\
\hline & & L3 system & $\begin{array}{l}1 \text { TV channel } \\
+600 \text { speech channels }\end{array}$ & 4 miles \\
\hline \multicolumn{2}{|c|}{$\begin{array}{l}\text { Single wire } \\
\text { transmission line }\end{array}$} & $\begin{array}{l}\text { About } \\
100 \text { to } 1,000 \mathrm{Mc} / \mathrm{s}\end{array}$ & $\begin{array}{l}\text { Probably a } \\
\text { few TV channels }\end{array}$ & About 2 to 10 miles \\
\hline \multirow{6}{*}{\multicolumn{2}{|c|}{ Microwave links }} & Below $500 \mathrm{Mc} / \mathrm{s}$ & Maximum of 60 & 40 to 50 miles \\
\hline & & 500 to $1,000 \mathrm{Mc} / \mathrm{s}$ & Up to 120 & 40 to 50 miles \\
\hline & & $2,000 \mathrm{Mc} / \mathrm{s}$ & $240 \times 6$ or $1 \mathrm{TV} \times 6$ & 30 to 40 miles \\
\hline & & $4,000 \mathrm{Mc} / \mathrm{s}$ & $600 \times 6$ or $1 \mathrm{TV} \times 6$ & 25 to 30 miles \\
\hline & & 6,000 to $8,000 \mathrm{Mc} / \mathrm{s}$ & $\begin{array}{l}600 \times 6 \text { or } 1 \mathrm{TV} \times 6 \\
\text { Maximum } 2 \mathrm{TV} \times 6\end{array}$ & 25 to 30 miles \\
\hline & & $11,000 \mathrm{Mc} / \mathrm{s}$ & Less than 600 & Less than 20 miles \\
\hline \multicolumn{2}{|c|}{$\begin{array}{l}\text { Long haul waveguide } \\
\qquad\left(\mathrm{H}_{\mathrm{Ol}}\right)\end{array}$} & $\begin{array}{l}30,000 \mathrm{Mc} / \mathrm{s} \\
\text { up to about } \\
100,000 \mathrm{Mc} / \mathrm{s}\end{array}$ & $\begin{array}{l}\text { 1, 000 super channels } \\
\equiv \text { several hundreds } \\
\text { of TV channels } \\
\equiv \text { several hundred } \\
\text { thousands of } \\
\text { speech channels }\end{array}$ & $\begin{array}{l}20 \text { to } 40 \text { miles } \\
\text { (attenuation } 2 \text { to } \\
4 \mathrm{db} / \text { mile) }\end{array}$ \\
\hline
\end{tabular}


derive the advantage of regeneration. Regeneration is a necessary requirement because of the large signal distortion, even with waveguides of moderate length (say 10 miles). It has been established beyond any doubt that for long-distance communication purposes only modulation systems capable of regeneration can be used, and of these PCM has definite technological advantages.

This paper is not an attempt at a balanced summary of the subject matter, but is a presentation of the opinions of the authors.

\section{Summary of Properties of Waveguides}

There are four principal factors influencing the performance of a waveguide: (1) Its geometry both in shape and size (in terms of the wavelength), (2) its mode of operation, (3) the value of the surface impedance of its walls, and (4) the nature and magnitude of the tolerances on the cross section.

For long-distance communication waveguides of circular cross section operating in the $H_{01}$ mode have been chosen. The choice is chiefly a matter of technological preferences based on a number of considerations. Such waveguides have relatively low dispersion and low attenuation and can be constructed to discriminate against unwanted modes, and the requirements on the tolerances on the cross section are less exacting than for other waveguides and modes.

The exact waveguide properties and construction is a matter of compromise between a number of conflicting factors such as cost of terminal equipment and repeaters, permissible signal distortion, service reliability, etc. And, since these factors can be traded for each other it is impossible to give a specification for a waveguide under any given conditions except in the light of accumulated experimental experience. But, to fix orders of magnitude a waveguide operating in the 4 - to 8 -mm band would be about 2 to 3 in. in diameter.

With waveguides there are two principal sources of signal distortion: (1) Delay distortion [6], and (2) mode conversion-reconversion phenomena [1, 4]. Delay distortion arises (like with most communication media) because of the nonlinearity of the phase characteristic, $\beta(\omega)$. This leads to the undesirable feature that the high frequency components of the signal travel faster than the low frequency components, a phenomenon known as dispersion. A corresponding distortion due to the variation in the attenuation characteristic $\alpha(\omega)$ is, with waveguides, negligible [6].

The actual bandwidth limitation due to delay distortion depends to a large extent on the modulation method and is particularly troublesome with FM. PCM, on the other hand, is rather immune, but even here $10-\mathrm{m} \mu \mathrm{sec}$ modulation pulses would be unrecognizable after a journey down a typical waveguide, say, 30 miles long; regeneration is, therefore, called for. In addition, certain amount of equalization of the $\beta(\omega)$ characteristic may be a necessity.
Mode conversion-reconversion is a new phenomenon, peculiar to waveguide transmission systems. It arises because a typical waveguide suitable for long distance transmission can support a large number of modes apart from the desired one $\left(H_{01}\right)$, and because of the irregularities in the waveguide (small dents, changes in diameter and cross sectional shape, small bends and kinks, etc.). In consequence, at each minute irregularity the preferred transmission mode $\left(H_{01}\right)$ becomes scattered and partially transformed into the parasite modes. The process is known as mode conversion and leads to a gradual diffusion of energy into the parasite modes and, therefore, increased attenuation. With some exceptions this process of mode conversion is actually not particularly harmful. It cannot, however, exist on its own but, by reciprocity, it is accompanied by the complementary process of "reconversion." In this process, the energy now partially carried in the unwanted modes becomes reconverted (by being scattered by subsequent irregularities) back to the $H_{01}$ mode. But since the group velocities of the parasite modes differ from that of the $H_{01}$ mode, the reconversion is not in phase with the signal carried in the $H_{01}$ mode. Further, since the degree of the distortion is phase dependent and the phase of the reconverted signal is related to the distance between the conversion and reconversion points in terms of the wavelength, the whole process of mode conversion-reconversion is frequency sensitive. The overall frequency attenuation curve is, therefore, very irregular, Jeading to considerable signal distortion. Because of the nature of mode conversionreconversion phenomena it is inconceivable that suitable terminal equipment could be constructed to counteract its effects. Mode conversion-reconversion effects are conveniently minimized by suitable waveguide design.

There is one particular aspect of mode conversionreconversion that calls for special attention: this arises through bending of the waveguide. The circular waveguide carrying the $H_{01}$ mode can support simultaneously the $E_{11}$ mode, having identical velocity. ${ }^{3}$ In a straight waveguide this is of no direct consequence, but if the waveguide is bent in an arc of a circle then the two modes become coupled with subsequent exchange of energy between the modes. This leads to large attenuation and signal distortion. Once again the effect is counteracted by judicious waveguide design.

It is possible to design a whole series of components [1,5] such as specially tailored bends and corners, tapers, mode transducers, atc. But the design is not as straightforward as with components for conventional single mode rectangular wavegujde. The waveguide can support a large number of modes and, therefore, any changes of the waveguide geometry must be made with great care bearing in mind mode conversion-reconversion phenomena. This requires a detailed study of wave transmission

${ }^{3}$ Such modes are termed degenerate. 
in multimode waveguides and the coupling effects due to irregularities; a field relatively unexploited in its analytical and experimental aspects.

Despite all the planning difficulties, a waveguide when properly designed will give satisfactory service and will handle successfully a bandwidth well in excess of $20 \mathrm{kMc} / \mathrm{s}$, which is equivalent to an intelligence bandwidth of about $1,000 \mathrm{Mc} / \mathrm{s}$, a capacity not offered by any other existing communication medium.

\section{Delay Distortion in Uniform Waveguides}

As is well known, with waveguides both $\alpha(\omega)$ and $\beta(\omega)$ are functions of frequency. It can be shown [6], however, that the effect of $\alpha(\omega)$ on signal distortion is negligible in comparison with $\beta(\omega)$. Here, therefore, only the effect of the $\beta(\omega)$ characteristic will be examined.

The phase propagation coefficient $\beta(\omega)$ is given by

$$
\beta=\sqrt{ }\left(\omega^{2}-\omega_{c}^{2}\right) / c,
$$

where $c$ is the velocity of light, and $\omega_{c}$ the cutoff frequency of the waveguide, which is a geometrical parameter. These relations are illustrated in figure 2 .

The phase $\left(v_{p h}\right)$ and group velocities $\left(v_{g}\right)$ are defined by

$$
\frac{v_{p h}}{c}=\frac{\omega_{o}}{\sqrt{\left(\omega_{o}^{2}-\omega_{c}^{2}\right)}}=\frac{c}{v_{g}} .
$$

These quantities are frequency dependent.

If the signal is composed of several frequencies then, naturally, the signal will be distorted because the high frequency components will travel faster I han the low frequency ones. The differential phase delay between the extreme components of the modulating envelope is a measure of signal distortion: it is the delay distortion term. For a waveguide operated at a frequency substantially above

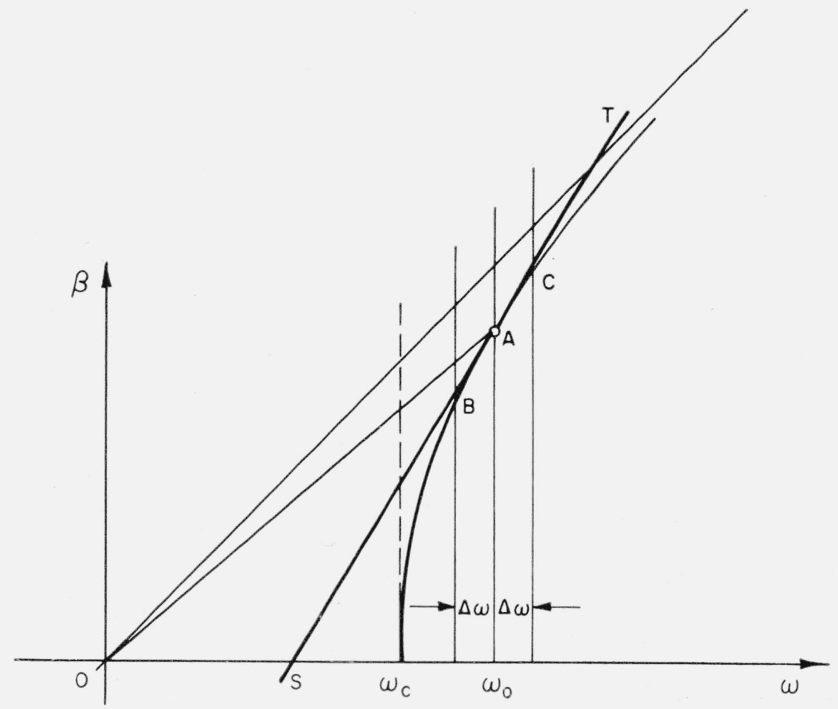

FIGURE 2, $\beta-\omega$ characteristic of a perfect waveguide. cutoff the bandwidth (in Mc/s) can be expressed as follows [6]:

$$
\mathrm{f}=(19 / F)\left(f_{o} / l\right)^{1 / 2}(\delta \phi)^{1 / 2}
$$

where

$\mathrm{F}=$ cutoff frequency/carrier frequency

$l=$ length of waveguide in kilometers

$f_{o}=$ carrier frequency in kilomegacycles per secona $\delta \phi=$ permissible phase delay between the carrier and the sideband in radians.

It has been assumed that $f$ is a small proportion of carrier frequency.

For example, for a typical rectangular waveguide $F=0.6$, and therefore at the carrier frequency of $10 \mathrm{kMc} / \mathrm{s}$ (and length $30 \mathrm{~km}$ ) we get

$$
f=9.4(1 \mathrm{rad})^{1 / 2} \mathrm{Mc} / \mathrm{s} \text {. }
$$

The permissible amount of delay distortion $(\delta \phi)$ is a function of modulation method. Clearly, with $\mathrm{AM}$ if $(\delta \phi)=\pi / 2$ then inversion of sidebands takes place: a condition of severe distortion. For AM, FM, and many other modulation methods the maximum possible value of $\delta \phi$ is only a small fraction of 1 rad. For PCM, however, $(\delta \phi)$ can take quite substantial values; once again, therefore, PCM is superior to the more common modulation methods.

For wideband application the modulation methods must be analyzed individually. For example, with pulse transmission systems, the energy contained in a pulse of finite duration gradually becomes spread out on the time scale. For a given waveguide length the degree of pulse distortion is a function of pulse duration. But up to a certain minimum pulse duration the pulse is quite recognizable and most of the energy is contained in the time interval equal to the duration of the transmitted pulse. For pulses of shorter duration the pulse distortion becomes quite severe and increases as pulsed duration decreases [6]; simultaneously the energy becomes diffused over a longer and longer time interval. The actual pulse width for which this happens is related to the geometry of the waveguide cross section and waveguide lengths (fig. 3).

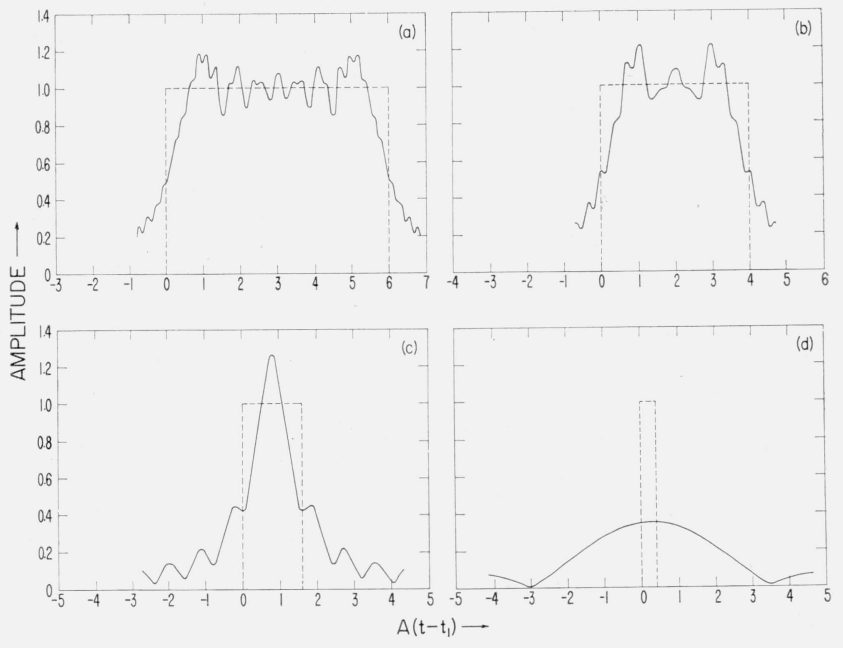

FIgure 3. Pulse distortion due to dispersion in waveguides. 
Typical waveguides for long-distance transmission have $F=0.15$, then at a frequency of $35 \mathrm{kMc} / \mathrm{s}$ pulses as short as $10 \mathrm{~m} \mu \mathrm{sec}$ can be transmitted without significant distortion over a distance of some $30 \mathrm{~km}$. Such performance is sufficient for many applications, but if it were required to transmit even shorter pulses some equalization of waveguide characteristic would then be necessary. Such equalization is best carried out at the terminal equipment.

\section{Properties of Uniform Waveguides and Their Uses}

\subsection{Simple Waveguides}

The reasons for choosing for long-distance transmission the circular waveguide operated in the circular electric mode $\left(H_{01}\right)$ have been explained in section 2. Such waveguides can support, apart from the desired $\left(H_{01}\right)$ mode a multitude of other modes all of which are undesirable. The total number of modes that can possibly propagate in a waveguide with diameter $D$ is known as the modulus of overmoding, $M$. This is approximately given by

$$
M=2.55\left(D / \lambda_{0}\right)^{2}
$$

The larger the modulus of overmoding, the larger the number of possible modes and, consequently, the greater the engineering difficulties in preventing mode conversion-reconversion phenomena. Yet, a large ratio $D / \lambda_{0}$ is necessary to have low attenuation and dispersion. Of necessity, therefore, the proportions of a waveguide are a compromise. Typically, $D / \lambda_{0}$ is of the order of 10 or more and the modulus of overmoding is, therefore, at least about 200 to 400 .

Each of the modes propagates with a characteristic group velocity given by

$$
v_{g} / c=c / v_{p h}=\sqrt{ }\left(1-F^{2}\right) .
$$

The commonest type of waveguide is a plain metallic pipe. With such waveguides the wall of the waveguide presents a surface resistance $R_{s}$ given by [7]

$$
R_{s}=\sqrt{ }(\pi f \mu / \sigma) \div \sqrt{ }\left(\mu_{o} / \epsilon_{o}\right)=R_{m}
$$

where $\sigma$ is the conductivity of the metal, $\mu$ its permittivity, and $\sqrt{ }\left(\mu_{o} / \epsilon_{o}\right)=377$ ohms, is the free space impedance. This surface resistance gives rise to attenuation which for $E_{m \bar{n}}$ modes is given by [7]

$$
\alpha_{m n}^{(e)}=\frac{8.686}{D / 2} \cdot \frac{R_{s}}{\sqrt{ }\left(1-F^{2}\right)},
$$

and for $H_{m n}$ modes by

$$
\alpha_{m n}^{(h)}=\frac{8.686}{D / 2} \cdot \frac{F^{2}}{\sqrt{ }\left(1-F^{2}\right)}\left[1+\frac{1}{F^{2}} \frac{\left(m / \chi_{m n}^{(h)}\right)^{2}}{1-\left(m / \chi_{m n}^{(h)}\right)^{2}}\right] R_{s} .
$$

These attenuations are given in decibels per meter if $D$, the diameter of the waveguide, is measured in meters.

The attenuation of the $H_{01}$ mode (the wanted mode) is given by [7]

$$
\alpha_{0}=\frac{8.686}{D / 2} \cdot \frac{F^{2}}{\sqrt{ }\left(1-F^{2}\right)} R_{m}
$$

Thus for a copper waveguide at the free space wavelength of $8.7 \mathrm{~mm}$, it is $1.2 \mathrm{db} / \mathrm{mile}$.

Most of the unwanted modes have attenuation and group velocities differing substantially from the $H_{01}$ mode. Thus attenuations of 10 to 50 times that of the $H_{01}$ mode is common and group velocities of most modes differ from the $H_{01}$ mode by as much as 10 percent or more. A few of the modes, however, have properties similar to those of the $H_{01}$ mode. For example, the $E_{11}$ mode has group velocity almost equal to that of the $H_{01}$ mode and a few of the modes, notably the $H_{02}, H_{03}, H_{12}, H_{13}, H_{22}$, and $H_{23}$, have attenuations only 3 to 10 times as much as the $H_{01}$ mode. These modes, therefore, tend to be particularly troublesome and the only way to deal with them satisfactorily is through judicious use of special waveguides.

\subsection{Special Waveguides}

Waveguides of special construction are necessary, as explained in the earlier sections, principally to combat or at least to minimize the mode conversionreconversion effects. For such applications, disk waveguides were first to be suggested and various elliptic, corrugated, and more complex structures were tried for negotiating bends. After many years of research it is now clear that only helical, and dielectric coated waveguides are likely to be of any extensive use. Such waveguides behave as if their surface impedance were anisotropic.

The theory of propagation in anisotropic waveguides can be quite involved, but since waveguides for long distance transmission have, in general, small surface impedance an approximate treatment is adequate, and this will be given here $[8,9]$.

The propagation coefficient in such waveguides is given by

$$
\gamma=\gamma_{o}+\delta \gamma=j \beta_{o}+(\alpha+j \delta \beta)=\alpha+j \beta,
$$

where the quantity $\delta \gamma(=\alpha+j \delta \beta)$ is composed of the attenuation coefficient $(\alpha)$ and a quantity $\delta \beta$ modifying the phase change coefficient $\left(\beta_{0}\right)$ of the waveguide with zero surface impedance. The coefficient $\alpha$ is proportional to surface resistance $\left(R_{s}\right)$ and $\delta \beta$ to surface reactance $\left(X_{s}\right)$, these being respectively the real and imaginary parts of surface impedance, $Z_{s}$. 
With anisotropic waveguides the quantity $Z_{s}$ may be described in terms of the matrix of its components; most simply in terms of its two principal components $Z_{\eta}$ and $Z_{\zeta}$ which are surface impedance components along the two principal axes. The sum of the projection of these components along the $\phi$ and $z$ directions define the circumferential $\left(Z_{\phi}\right)$ and axial $\left(Z_{z}\right)$ components of the surface impedance; and it is these components that are used in the subsequent formulas. $\quad Z_{\phi}$ can be regarded as the surface impedance presented to the currents flowing in the waveguide wall in the circumferential direction and $Z$, a corresponding quantity for currents flowing in the axial direction.

In such waveguides the attenuation of $E$-waves is given by [9]

$$
\alpha^{(e)}=2 R_{z} / D \sqrt{ }\left(1-F^{2}\right) \quad \text { nepers }
$$

and for $H$-waves by

$$
\begin{aligned}
\alpha_{m n}^{(h)}=\frac{2}{D} \frac{F^{2}}{\sqrt{\left(1-F^{2}\right)}}[ & \left.R_{\phi}+R_{z} \frac{1-F^{2}}{F^{4}}\left(\frac{m}{\pi} \frac{\lambda_{o}}{D}\right)\right] \\
\times & {\left[1-\left(\frac{m}{\pi} \frac{\lambda_{o}}{D}\right)^{2}\right]^{-1} \text { nepers } }
\end{aligned}
$$

where the meaning of symbols is as before.

In the above formulas if $X_{z}, X_{\phi}$ are substituted for $R_{z}$ and $R_{\phi}$, respectively, then $\delta \beta$ will be obtained.

\subsection{Dielectric Coated Waveguides}

A metallic surface exhibits a surface impedance given by [8]

$$
Z_{m}=(1+j) R_{m}
$$

where $R_{m}$ is given by (6). The surface of a copper tube $\left(\sigma=5.8 \times 10^{7} \mathrm{mho} / \mathrm{m}\right)$, therefore, presents at $\lambda_{0}=8.7 \mathrm{~mm}$ a surface impedance of $(1+j) \times 1.28 \times$ $10^{-4}$, relative to free space (about $0.048 \mathrm{ohms}$ ).

If, now, the metallic tube is coated with a thin layer of dielectric then its surface impedance will be enhanced as given in table 2 , where $t$ is the thickness of the dielectric layer, $k_{o}=2 \pi / \lambda_{o}, \epsilon_{r}$ relative permittivity, and $\delta$ the loss angle of the dielectric. Clearly, by choosing suitable materials and the thickness of the dielectric layer, it is possible to obtain within wide limits any required surface impedance compo-

TABLE 2. The surface impedance of a dielectric coated metal

\begin{tabular}{|c|c|c|}
\hline $\begin{array}{c}\text { Surface } \\
\text { impedance }\end{array}$ & Re part & Im part \\
\hline $\begin{array}{l}Z_{z}=R_{2}+j X_{2} \\
Z_{\phi}=R_{\phi}+j X_{\phi}\end{array}$ & $\begin{array}{l}R_{2}=t k_{o}\left(\tan \delta / \epsilon_{\tau}\right) \\
R_{\phi}=\frac{1}{3}\left(t k_{o}\right)^{3}\left(\epsilon_{r} \tan \delta\right)\end{array}$ & $\begin{array}{l}X_{z}=t k_{o}\left(1-1 / \epsilon_{\tau}\right) \\
X_{\phi}=\frac{1}{3}\left(t k_{o}\right)^{3}\left(\epsilon_{\tau}-1\right)\end{array}$ \\
\hline
\end{tabular}
surface

These value must be augmented by $Z_{m}=R_{m}+j X_{m}\left(R m=X m=\sqrt{\pi f \mu / \sigma} \sqrt{\left.\epsilon_{o} / \mu_{0},\right)}\right.$ the surface impedance of the metal. nents and, consequently, the attenuation and phase velocity of the $E$ and $H$ modes can be varied relative to the $H_{\text {on }}$ modes. By way of illustration, at the free space wavelength of $8.7 \mathrm{~mm}$, for $\epsilon_{\tau}=2$, $\tan \delta=0.15$, and $t=0.046 \mathrm{~mm}$, the surface impedance components become $Z_{z}=2.5 \times 10^{-3}+j 1.7 \times 10^{-2}$ and $Z_{\phi}=3.7 \times 10^{-6}+j 3.3 \times 10^{-4}$ over and above the value of $Z_{m}$. If we substitute these values in eqs (11) and (12) we find that for a waveguide of $7-\mathrm{cm}$ diam, there is a 20-fold increase in attenuation of $E$ and $H$ modes (other than $H_{o n}$ ) relative to the $H_{01}$ at the expense of only a 3 percent increase in attenuation of the $H_{01}$ mode. The surface reactance $X_{z}$ is, at the same time, increased by a factor of 140 leading to a substantial change in phase velocity of most modes.

The increase in relative attenuation is necessary to cope with mode conversion-reconversion, due to irregularities in the waveguide, while the increase in the surface reactance, $X_{z}$, is necessary so that the waveguide can cope with bends.

\subsection{Ring or Disk Structures}

Any periodic array of coaxial disks or rings of different surface impedances falls into this class. If the pitch of such a structure is small in comparison with the wavelength then a simple approximate treatment is possible, as follows:

Let $Z_{1}$ and $Z_{2}$ be the surface impedance of the individual elements, thickness $t_{1}$ and $t_{2}$, respectively, then the anisotropic components of the effective surface impedance are given by

$$
\left.\begin{array}{l}
Z_{2}=\frac{Z_{1} t_{1}+Z_{2} t_{2}}{t_{1}+t_{2}} \\
Z_{\phi}=\frac{Z_{1} Z_{2}\left(t_{1}+t_{2}\right)}{Z_{2} t_{1}+Z_{1} t_{2}}
\end{array}\right\} .
$$

Since in most applications $\left|Z_{2}\right|>\left|Z_{1}\right|$ and $t_{2}<<t_{1}$ these expressions can be further simplified to

$$
\left.\begin{array}{l}
Z_{z}=Z_{2} t_{1} /\left(t_{1}+t_{2}\right) \\
Z_{\phi}=Z_{1}\left(t_{1}+t_{2}\right) / t_{1}
\end{array}\right\} .
$$

Thus given $Z_{1}, Z_{2}, t_{1}$, and $t_{2}$ the surface impedance components $Z_{z}$ and $Z_{\phi}$ can be calculated. In turn, the attenuation and phase propagation coefficients can be obtained from (11) and (12).

The quantities $Z_{1}$ and $Z_{2}$ are determined from the knowledge of the actual physical structure. This can be a lengthy calculation, but since in practical applications only an estimate of the value of the surface impedance is needed, it is permissible to make a number of rather drastic approximations. For example, for a corrugated waveguide (fig. 4), $Z_{1}$ is the copper intrinsic impedance and $Z_{2}$ is the input impedance to a parallel plane transmission line, 


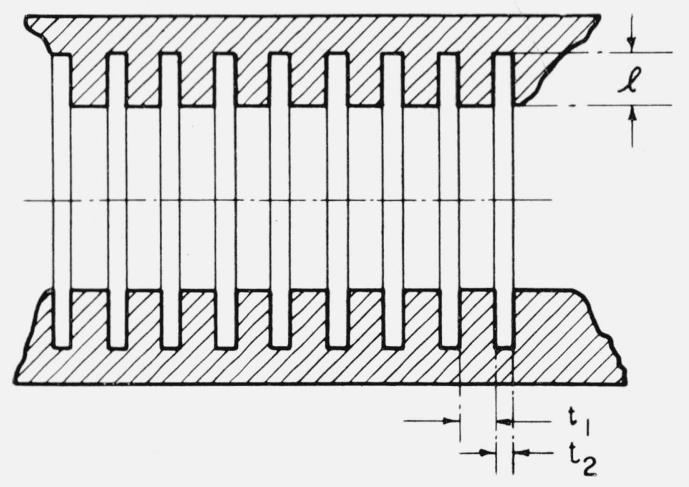

Figure 4. Corrugated waveguide.

length $l$, and separation between plates $t_{2}$. Thus

$$
\left.\begin{array}{l}
Z_{z}=R_{m}\left[1+l /\left(t_{1}+t_{2}\right)\right]+i 2 \pi \frac{l}{\lambda_{0}} t_{2} /\left(t_{1}+t_{2}\right) \\
Z_{\phi}=Z_{m}\left(t_{1}+t_{2}\right) / t_{1}
\end{array}\right\}
$$

where $Z_{m}$ is the surface impedance of the metal (e.g., eq (13)).

It will be observed that $Z_{\phi}$ has been increased a little, in proportion of $\left(t_{1}+t_{2}\right) / t_{1}$, but that $Z_{z}$ is highly reactive. Such waveguides are particularly suitable for negotiating bends.

Clearly, other structures can be calculated in an analogous manner.

\subsection{Helical Waveguides}

It will be observed from eqs (15) that $Z_{z}$ and $Z_{\phi}$ can be adjusted within very wide limits to any desirable values provided that the proportions of the microstructure of the waveguide surface are suitably chosen. This feature makes the disk waveguides, and their variants, extremely attractive. But, unfortunately, such waveguides are difficult if not impossible to manufacture in quantity. It is for this reason that helical waveguides are preferred.

It can be shown that if the pitch of the helical waveguide is very small, as would be with waveguides wound with a fine wire, and the surface impedance not too large (say less than $10^{-1}$, i.e., some $40 \mathrm{ohms}$ ), then the helical waveguide behaves as a corresponding ring structure.

A particularly important structure is shown in figure 5; this shows a helix of insulated (copper) wire set in a suitable resin and backed by a resistive layer of surface impedance $R$, the whole structure is enclosed in a suitable protective jacket. If the wire diameter is $t_{1}$ and the gap between the wires $t_{2}$, then provided that $t_{2}$ is not too small, the structure behaves approximately as a corresponding disk structure (fig. 6) with $l=t_{1}$.

Using the procedure as outlined before, eqs (15) can be used where $Z_{1}=Z_{m}=$ copper intrinsic impedance and $Z_{2}$ is taken equal to the input impedance of a transmission line length $l$, terminated in a

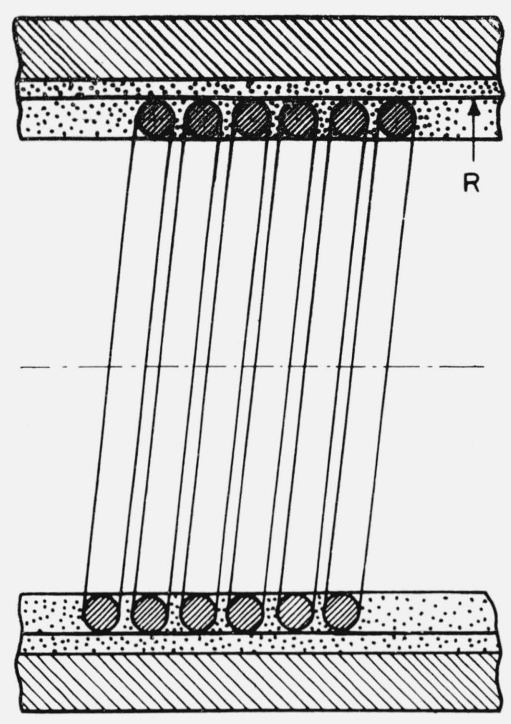

Figure 5. A helical waveguide.

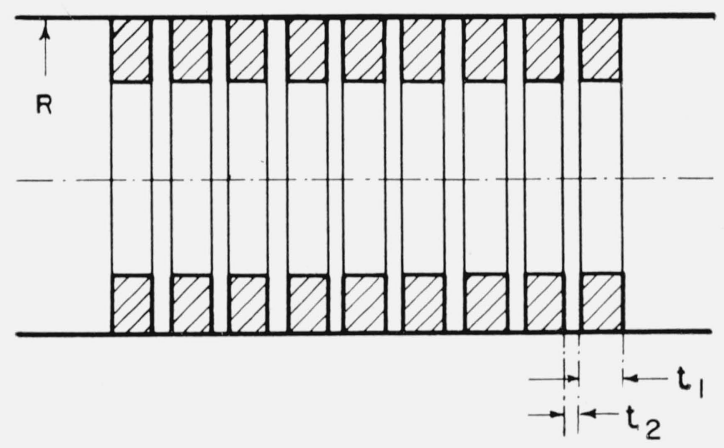

Figure 6. Ring structure with a resistive layer.

resistive impedance $R$. Clearly, therefore,

$$
\left.\begin{array}{l}
Z_{z}=R \frac{t_{2}}{t_{1}+t_{2}}+j 2 \pi\left(l / \lambda_{0}\right) \frac{t_{2}}{t_{1}+t_{2}} \\
Z_{\phi}=Z_{m} \frac{t_{1}+t_{2}}{t_{1}}
\end{array}\right\} .
$$

As a numerical example consider a helical waveguide with following constants: $t_{1}=l=\lambda_{0} / 10, \quad t_{2}=\frac{1}{2} t_{1}$, $R_{m}=10^{-4}$ (approximately copper impedance), $R=10^{-2}$ (approximately $4 \mathrm{ohms}$ ) then, neglecting the lay angle of the helix, at a free space wavelength of $8.7 \mathrm{~mm}$ the surface impedance components become

$$
\begin{aligned}
& Z_{z}=0.003+j 0.021 \\
& Z_{\phi}=1.5(1+j) \times 10^{-4} .
\end{aligned}
$$

The propagation coefficients follow from eqs (7) and (8). Thus for a waveguide $7 \mathrm{~cm}$ in diam there is a 50 percent increase in the attentuation of the $H_{01}$ 
mode, and a 30-fold increase in the attenuation of most $E$-modes. The attenuation of the lower order $H$-modes has also been increased by a factor of 30 , while the surface reactance is increased 200 times.

\section{Basic Theory of Nonuniform Waveguides}

Theory of nonuniform waveguides is fundamental for thorough understanding of transmission aspects as well as design of many waveguide components peculiar to long distance transmission by waveguide. Thus, commonly, waves are conveyed from an oscillator via a single mode rectangular waveguide through a transducer to a circular waveguide then through a taper to the main waveguide run.

We shall see that a mode transducer, a bent waveguide, as well as a taper are particular embodiments of nonuniform transmission lines. The main waveguide run is also a nonuniform transmission line in the sense that it can carry a large number of modes and all minute irregularities act as coupling elements between the lines; each line representing one particular mode.

In general, we represent a nonuniform waveguide by its equivalent circuit: $n$ coupled transmission lines, where $n$ is the number of possible modes of propagation (see fig. 7).

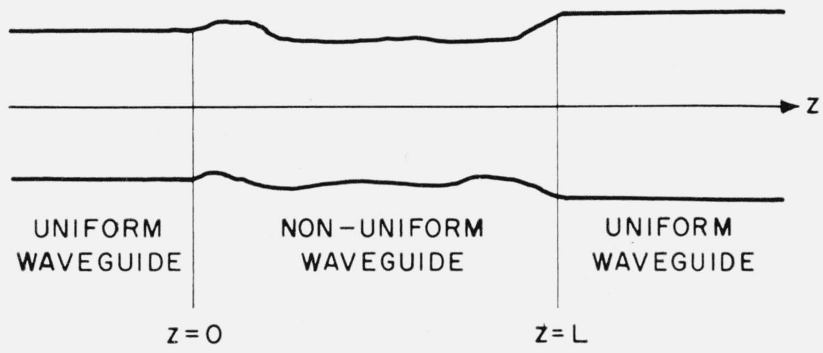

FIGURE 7. Schematic of a nonuniform waveguide.

For convenience in analysis, nonuniform waveguides can be subdivided into (1) straight waveguides, and (2) bent waveguides.

\subsection{Straight Waveguides}

The first general solution to transmission in nonuniform waveguides was obtained by Stevenson [10] in terms of a series of transverse wave functions. Through such an approach it is possible to obtain equivalent generalized telegraphist's equations [11] of the waveguide. Adopting Reiter's approach $[12,13]$, it can be shown [17] that for the forward and backward traveling waves the following equa- tions hold

$$
\begin{aligned}
& \frac{d A_{i}^{+}}{d z}=-j \beta_{i} A_{i}^{+}-\frac{1}{2} \frac{d\left(\ln K_{i}\right)}{d z} A_{i}^{-}+\sum_{p}\left(S_{i_{p}}^{+} A_{p}^{+}+S_{i_{p}}^{-} A_{p}^{-}\right), \\
& \frac{d A_{i}^{-}}{d z}=-\frac{1}{2} \frac{d\left(\ln K_{i}\right)}{d z} A_{i}^{+}+j \beta_{i} A_{i}^{-}+\sum_{p}\left(S_{i_{p}}^{-} A_{p}^{+}+S_{i_{p}}^{+} A_{p}^{-}\right)
\end{aligned}
$$

where $z$ is the coordinate along the waveguide axis.

$A_{i}^{ \pm}$are amplitude coefficients of the forward $(+)$and backward (-) traveling waves on the $i$ th line (corresponding to the $i$ th mode).

$S_{i p}^{+}$are the corresponding coupling coefficients between the waves on lines $i$ and $p$ (a parameter related to the geometry of the waveguide).

$K_{i}$ is the wave impedance of the $i$ th line.

$\beta_{i}$ the propagation coefficient.

The above are exact equations for a set of lossless nonuniform transmission lines where $S, K$, and $\beta$ are, in general, functions of $z$. These equations are exact representation of transmission in a nonuniform waveguide. In principle, therefore, given the functional dependence of the waveguide geometry on the coordinate $z$, the propagation problem can be solved, but the computational difficulties are in most cases formidable. With practical waveguides, however, it is possible to proceed with a number of simplifying assumptions leading in many cases to rather simple solutions $[14,15,16,17]$.

In the first place, we assume that the waveguide geometry changes slowly and consequently $S, K$, and $\beta$ are slowly varying functions of $z$. The coupling between the lines per unit length will therefore be small. If now the input at one end of the waveguide is a pure mode, amplitude $A_{o}$, then the boundary conditions are simply

$$
\left.\begin{array}{ll}
A_{m}^{+}(0)=A_{o} & A_{m}^{-}(L)=0 \\
A_{i}^{+}(0)=0 & A_{i}^{-}(L)=0 \quad(i \neq m)
\end{array}\right\} .
$$

With these assumptions only the coupling between the line $m$ and the remaining lines need be considered; the cross coupling between the lines can be neglected and so can the reflected waves. We then obtain a simplified solution in the form [17]

$$
A_{m}^{+}(z)=A_{o} \exp \left[-j \int_{0}^{z} \beta_{m} d z\right]
$$

The amplitude of the remaining waves are given by

$$
\left.\begin{array}{l}
A_{m}^{-}(0)=-\exp \left[-j \int_{0}^{L} \beta_{m} d z\right] \int_{0}^{L}\left(S_{m m}^{-}-\frac{1}{2} \frac{d\left(\ln K_{m}\right)}{d z}\right) \exp \left[-j 2 \int_{0}^{Z} \beta_{m} d z\right] d z \\
\begin{array}{c}
A_{i}^{+}(L) \\
A_{i}^{-}(0)
\end{array}= \pm \exp \left[-j \int_{0}^{L} \beta_{i} d z\right] \int_{0}^{L} S_{i m}^{ \pm} \exp \left[-j \int_{0}^{z}\left(\beta_{m} \mp \beta_{i}\right) d z\right] d z
\end{array}\right\}
$$


In the derivation of these expressions it has been assumed that none of the modes are at the cutoff. When dealing with components, such as tapers for example, some modes are of necessity at the cutoff at some cross section along the taper, and it would appear that (20) and (21) are not a sound basis for design. Nevertheless, it appears that the use of (20) and (21) is usually justified [18].

It is to be observed that usually it is only the lower order modes that need to be at all considered (typically $H_{02}, H_{03}, H_{12}, H_{13}, H_{22}, H_{23}$; cf., section 4.1.).

The $\beta$ coefficients of these modes differ but very little from that of the $H_{01}$ and consequently (eq (21)) only the coefficients of the forward traveling waves $\left(A^{+}\right)$need be considered; the coefficients $A^{-}$are very much smaller and can, therefore, be neglected.

Using eqs (21) an interesting proposition can be proven. In general, a pure mode at the input to a waveguide will produce at the output a combination of modes. For a good transducer the output consists of an almost pure mode of the desired kind. The purity of this mode can now be made as high as desired merely by increasing the length of the transducer: the longer and more gradual the transducer the purer is the mode at the output.

\subsection{Propagation in Bent Waveguides}

The general coupled transmission line equations for a bent waveguide of arbitrary cross section were given by Shimizu [19] from which the relations for a circular waveguide follow. The particular case of a bent circular waveguide was analyzed earlier by Jouguet [20], who analyzed the field in a curved waveguide by means of perturbation calculus and obtained the field in terms of an expansion in powers of $1 / R$, where $R$ is the radius of curvature of the waveguide.

It can be shown that if a curved section of waveguide is interposed between two straight waveguides (fig. 8) and a pure $H_{01}$ mode is incident at one end of the curved waveguide, then the wave appearing at the other end is composed of the $H_{01}, E_{11}$, and a series of $H_{1 \mathrm{n}}$ modes [19, 20,21], but the exact proportion

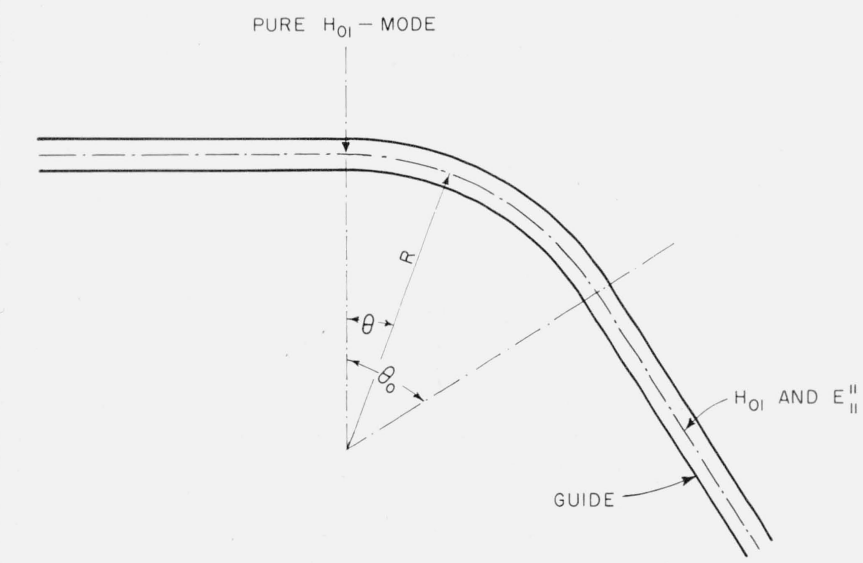

Figure 8. Curved circular waveguide. of each of the modes depends on the geometry of the bend. The equivalent circuit of the bend is therefore $n+2$ coupled transmission lines : one for the $H_{01}$, one for the $E_{11}$, and $n$ lines one for each of the $H_{1 n}$ modes. The coefficients of the coupling matrix are constants related to the geometry of the bend. But it can be shown, further, that if the radius of curvature is large, the series of the $H_{1 \mathrm{n}}$ modes may be neglected, since these are of $1 / R^{2}$ or higher order, and therefore only the $H_{01}$ and $E_{11}$ modes need be considered. The equivalent circuit is then, simply two coupled transmission lines.

Jouguet's analysis can be further extended to waveguides of arbitrary surface impedance [2]. It transpires that provided the radius of bend is not too small, the field inside a curved waveguide can be represented by ${ }^{4}$

$$
\Psi=A\left(\mathscr{H}_{01}+\tau \varepsilon_{11}\right) e^{-\gamma l}
$$

that is the field is a linear combination of the $H_{01}$ and $E_{11}$ modes in a definite proportion. The proportionality factor can take two possible values given by

where

$$
\tau^{2}-2 p \tau-2=0
$$

$$
p=\frac{h_{0} \beta_{0}}{k_{0}^{2}} \frac{R}{s} j Z_{s}
$$

If the surface impedance and the radius of curvature are small then $p$ is small and $\tau$ is approximately given by $\sqrt{2}$; therefore, the expression for the field [2] can be put into the form

$$
\Psi=\mathscr{H}_{01} \cos \delta \beta_{R} l+j \sqrt{2} \varepsilon_{11} \sin \delta \beta_{R} l e^{-\gamma_{0} l},
$$

where

and

$$
\left.\begin{array}{rl}
\delta \beta_{R} & =\frac{k_{0}}{h_{0} R \sqrt{2}} \\
\gamma_{1} & =j\left(\beta_{0} \pm \delta \beta_{R}\right)+\frac{1}{2}\left(\alpha_{E}+\alpha_{H}\right)
\end{array}\right\}
$$

with $\beta_{0}$ the phase propagation coefficient of the straight waveguide, $\alpha_{E}$ and $\alpha_{H}$ the attenuation coefficients of the $E_{11}$ and $H_{01}$ modes in the straight waveguide.

Two important conclusions must be made: (1) The energy is carried alternately in the $H_{01}$ and $E_{11}$ modes, and (2) the mean attenuation of the complete normal mode (25) is equal to the mean of those for the $E_{11}$ and $H_{01}$ modes.

Evidently, the waveguide is unsuitable for the transmission of the $H_{01}$ mode around the bends. However, the examination of the above equations shows that if $p$ is a large real number a different situation arises. Large real values of $p$ are obtained for highly reactive surfaces ( $X_{s}$ a large number), and in such waveguides the two modes $H_{01}$ and $E_{11}$

4. $\mathscr{H}_{01}$ stands for the field of the $H_{01}$ mode, and $\varepsilon_{11}$ stands for the field of the 
propagate almost independently of each other, but the attenuation of $H_{01}$ mode is slightly increased. In such a waveguide, the attenuation of the $H_{01}$ mode is given by [2]

$$
\alpha_{1}=\alpha_{0}+\alpha_{R}
$$

where $\alpha_{0}$ is the attenuation in the straight waveguide and is given by (eq (12))

$$
\alpha_{0}=\frac{2}{D} \frac{F^{2}}{\sqrt{ }\left(1-F^{2}\right)} R_{\phi}
$$

The additional attenuation due to the bend is given by

$$
\alpha_{R}=\frac{D}{4 R^{2}} \frac{R_{s} /\left|Z_{s}\right|^{2}}{F^{2} \sqrt{ }\left(1-F^{2}\right)} .
$$

Clearly, by choosing a sufficiently large surface reactance it is possible to bring this increase in attenuation below any predetermined level. Such values of surface reactance can be conveniently obtained either by coating the waveguide surface with a layer of dielectric or by corrugating the waveguide surface in a suitable manner.

The ratio $\alpha_{R} / \alpha_{0}=\nu_{R}\left(R_{z} / R_{\phi}\right)$ defines a quality factor of the bent waveguide. With the help of figure 9 it is possible to determine the required value of surface reactance for a given value of radius of curvature and quality factor $\nu_{R}$. Clearly, helical waveguides of appropriate proportions (sec. 4) can be designed for the purpose of negotiating bends.

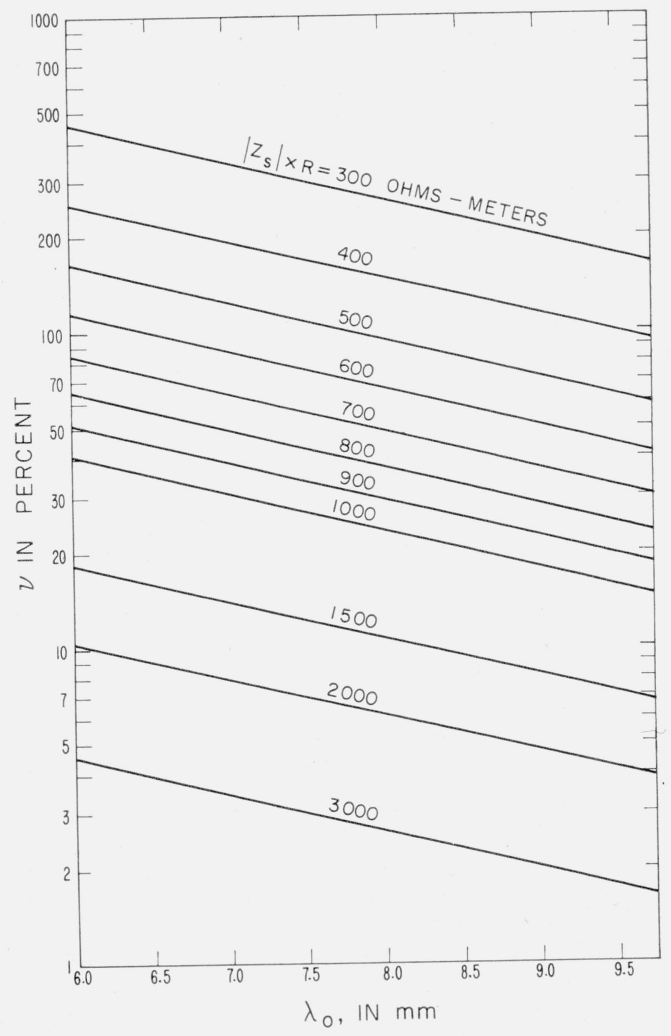

Figure 9. Quality factor, $\nu$, of a bent waveguide of 7-cm diameter as a function of wavelength, with $\left|\mathrm{Z}_{\mathrm{s}}\right| \mathrm{R}$ as parameter.
With suitably designed waveguides, bends even of a few hundred feet in radius can be negotiated quite successfully. A further improvement can be achieved by increasing gradually the radius of bend by means of a suitable transition curve [22] (a "tapered transition"). This is yet another embodiment of a set of nonuniform coupled transmission lines.

\section{Transducers}

A transducer is a waveguide configuration which achieves a required change of mode pattern and/or pattern size.

\subsection{Tapers}

A taper is the simplest illustration: it achieves a change in waveguide diameter and, if properly designed, leaves the mode pattern unaltered yet changed in size.

The rigorous theory $[23,24]$ of tapers can be very involved indeed but for our applications a number of approximations are permissible resulting in an adequate and simple design procedure.

Consider two coaxial waveguides joined by a conical taper and suppose that the larger waveguide (radius $a$ ) is operated substantially above cutoff (overmoded waveguide) and the smaller waveguide (radius $b$ ) carries a pure $H_{01}$ mode. The taper will, evidently, give rise to a series of unwanted modes besides the $H_{01}$ mode, but because of the symmetry of the structure only the $H_{o n}$ modes will be present. If, now, the diameter of the smaller waveguide is sufficiently small to permit only the $H_{01}$ mode to propagate, the amplitudes of the $H_{\text {on }}$ modes in the larger waveguide will be a simple function of the parameter $\sigma$ given by

$$
\sigma=\pi \frac{a}{\lambda} \frac{a-b}{L}
$$

where $L$ is the length of the taper and $\lambda$ the free space wavelength.

These relations are obtained from simple analytical considerations [25] by expanding the fields in the circular waveguides in cylindrical harmonics and the field in the conical region in spherical harmonics and equating the fields in the cross sections radius $a$ and $b$, respectively.

It can be shown that out of the modes produced by the taper the $H_{02}$ mode is largest in amplitude. Considering, therefore, the conversion into the $\mathrm{H}_{02}$ mode only we get for the taper length

$$
L \simeq \frac{0.57}{\sqrt{p}} \frac{a}{\lambda}(a-b)
$$

where $p$ is power conversion coefficient, which is the ratio of the permitted power in the $H_{02}$ mode to that in the $H_{01}$ mode.

For some applications the length of such a taper may be excessive, but a further improvement is possible by using a two-section conical taper (fig. 10). 


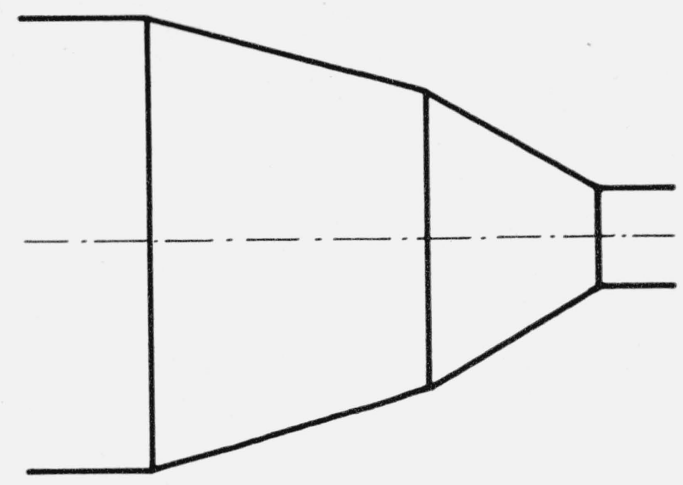

Figure 10. Schematic of a two-section conical taper.

For such a taper [26] by choosing correctly the proportions of the two cones the performance of the taper can be considerably improved. Optimum performance is possible only at one frequency, but by correct design it is possible to keep the sum of the energy carried in the $H_{02}$ and $H_{03}$ modes below any required level, over a substantial bandwidth [26].

More elaborate design procedures have been devised by several investigators [18, 24, 27]. But it appears [28] from results on antenna theory that provided the taper contour is "smooth and gradual" and that the derivatives at the taper ends are zero, there will be very little mode conversion even over a wide bandwidth.

\subsection{Mode Transducers}

With the exception of a few isolated instances there is no general design procedure for mode transducers. In a few simple cases it is possible to predict from the changes in the waveguide geometry the type of mode produced and with a little engineering intuition to design sometimes a reasonably satisfactory transducer. In most cases, however, even the intuitive approach is of no avail. Recently a satisfactory design procedure has been proposed [29] and confirmed experimentally. This is explained below.

Consider a mode transducer connecting two uniform waveguides $A$ and $B$. According to an established theorem, if the change from waveguide $A$ to waveguide $B$ is effected very gradually then a pure mode in waveguide $A$ will produce a substantially pure mode in waveguide $B$ : the purity of the mode in waveguide $B$ will be the higher the longer the transducer.

To determine the modes present at the ends of the transducer, it is necessary to choose several cross sections along its length and to determine the eigen functions in each. If the number of cross sections chosen is sufficient, then from the similarity between the successive field patterns of field distribution it is possible to examine the passage of any given mode along the whole length of the transducer, in detail.

To design a mode transducer, the desired modes in the uniform waveguides are given, and it is then required to determine the surface of the transducer for reasonable mode purity. We then choose a crosssectional wave function $\Psi$ (as a continuous function of $z$ ) such that it becomes the desired mode at each end of the transducer (at $z=0$ and $z=L$, where $L$ is the length of the transducer). This wave function determines the surface of the transducer; and, provided that the function $\Psi$ is a slowly varying function, the modes involved will be substantially pure. The design procedure is essentially numerical or graphical.

As an illustration consider the $H_{01}$ circular to $H_{01}$ rectangular mode transducer. For convenience and better illustration we propose to design the transducer in two parts: (1) $H_{01}$ circular to $H_{02}$ rectangular, and (2) $H_{02}$ rectangular to $H_{01}$ rectangular.

For the first transducer a convenient choice for the wave function is

$$
\Psi_{m}=g_{1}(z) J_{o}\left(\frac{\pi}{w} \sqrt{x^{2}+y^{2}}\right)+g_{2}(z) \cos \frac{\pi}{w} x
$$

where to satisfy the requirements at each end of the transducer, the conditions are

$$
\begin{array}{ll}
g_{1}(0)=1 & g_{1}\left(L_{1}\right)=0, \\
g_{2}(0)=0 & g_{2}\left(L_{1}\right)=1,
\end{array}
$$

with $L_{1}=$ the length of the transducer and $x, y=$ Cartesian coordinates in the transverse plane.

The diameter, $d$, of the circular waveguide and the width $2 w$ of the rectangular waveguide are adjusted so that their cutoff numbers are equal $(d / w=2.44)$.

Evidently the function $\Psi_{m}$ reduces at the ends of the waveguide to the eigenfunctions of the circular and rectangular waveguides respectively. The surface of the transducer for any intermediate values of $z$ is most easily determined graphically as follows. For any required value of $z$, curves of $\Psi_{m}=$ a constant are plotted: these represent the lines of electric field. But a permissible boundary must be normal to such lines and, therefore, the cross-sectional contour can be mapped. Figure 11 shows four contours of such a transducer, and since we know that the transducer must be gradual, in most cases four contours are sufficient to determine the surface of the transducer.

Since the wave functions of the $H_{02}$ and $H_{01}$ modes have the same mathematical formula the obvious choice for the wave function of the second transducer is [30]

$$
\Psi_{m}=\cos \frac{\pi}{w} x
$$

The curves $\Psi_{m}=$ a constant are simply straight lines and, therefore, the transducer has the shape shown in figures 12 and 13 ; the dimension $\delta$ changes slowly from $\delta=0$ to $\delta=h$.

Since $h$ is not equal to the diameter of the circular waveguide a further taper is necessary to connect the two transducers. Thus the complete $H_{01}$ circular to $H_{02}$ rectangular transducer is made up from the above three parts. 


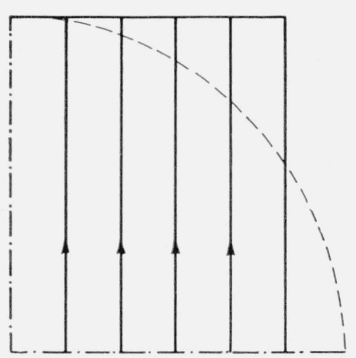

(c)

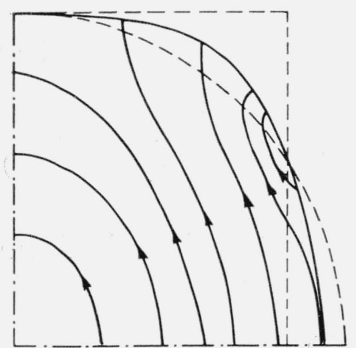

FIGURE 11. Schematic showing a gradual mode transformation from the $\mathrm{H}_{01}$ circular to the $\mathrm{H}_{02}$ rectangular (only one quadrant showing).

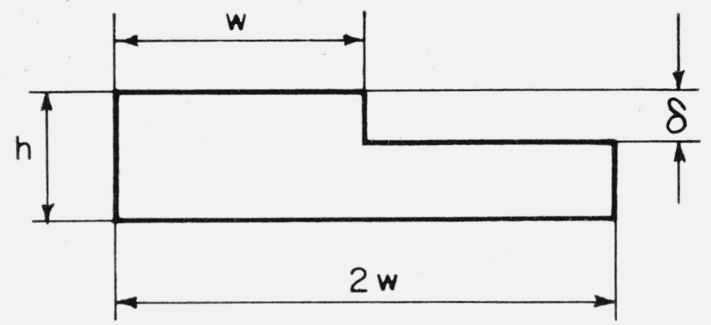

Figure 12. Cross section of an $\mathrm{H}_{01}$ to $\mathrm{H}_{02}$ rectangular transducer.

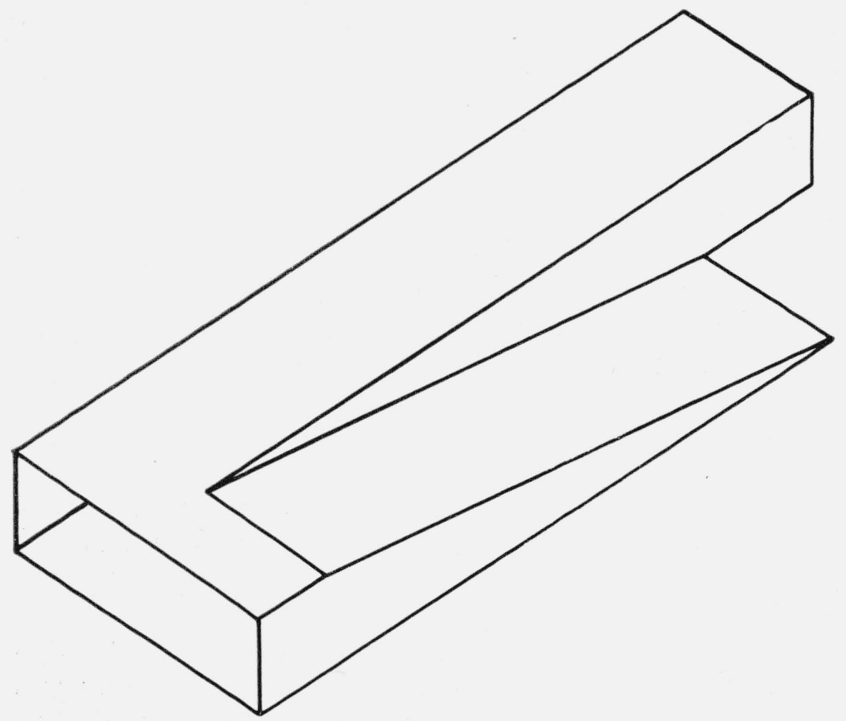

Figure 13. Perspective view of the complete $\mathrm{H}_{01}$ to $\mathrm{H}_{02}$ rectangular transducer.

\section{Slightly Irregular Waveguides}

The general problem of propagation in slightly nonuniform waveguides was investigated by Katzenelenbaum [31] and in a number of specific cases by Morgan [32] and Iiguchi [33].

In the case of a waveguide carrying an $H_{01}$ mode at a frequency substantially above cutoff, a quasioptical approach [34] can be adopted leading to simple derivation of relevant formulas. The problem can be stated with reference to figure 13 . The cross section of waveguide $A$ is circular and that of $B$ differs a little but in an arbitrary manner. By assuming that the field in the aperture is substantially the field as produced by the $H_{01}$ mode in the waveguide $A$ according to geometrical optics, simple integral relations can be derived for the intensity of the modes produced in waveguide $B$. There are only $H$-modes produced and the amplitude of the $H_{j}$ mode is given by [34]

$$
A_{j}=\int_{S_{0}} \nabla_{t} \Psi_{j} \nabla_{t} \Psi_{01} d S
$$

where $S_{o}$ is the aperture, $\nabla_{t}$ is the gradient operator in the transverse plane, $\Psi_{j}$ is the wave function of the $H_{j}$ mode in waveguide $B$, and $\Psi_{01}$ is the wave function of the $H_{01}$ mode in waveguide $A$.

In the case of a junction formed by two offset circular waveguides of identical diameters only $H_{1 n}$ modes will be generated if the exciting mode is a pure $H_{01}$ mode. If there is a small change in waveguide diameter and the waveguides are coaixal then only $H_{o n}$ modes are produced. Similar deductions can be made for other discontinuities without actually solving the expression (35).

For the two coaxial waveguides of slightly different diameter (see fig. 14) eq (35) leads to particularly simple relations. In this case the amplitude $\left(A_{o n}\right)$ of the $H_{\text {on }}$ mode produced by the incident $H_{01}$ mode is given by

$$
\frac{A_{o n}}{A_{01}}=\frac{2 \chi_{1} \chi_{n}}{\chi_{1}^{2}-\chi_{n}^{2}}\left(\frac{r_{2}-r_{1}}{r_{1}}\right)
$$

where $\chi_{i}$ is the $i$ th root of $J_{1}(x)=0$.

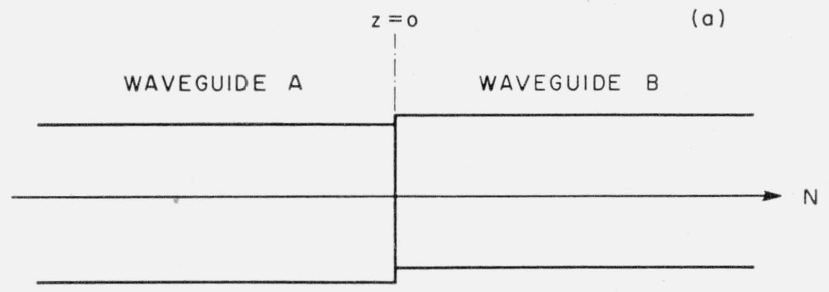

(b)

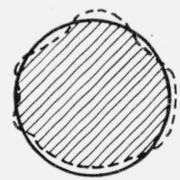

FIGURE 14. The junction of two waveguides of slightly different cross section. 
Consider now two such steps in tandem. Clearly the contributions from the conversions must be combined taking the correct phases into account. It can then be shown that if the inserted length of the waveguide is one-half beat wavelength (of $H_{01}$ and $H_{02}$ ) long and its diameter the mean of the two extreme waveguides, then the mode conversion is minimized [35]. The same procedure can be applied to other $H_{o n}$ modes and a suitable transition between two waveguides of different diameters designed.

\section{Mode Conversion-Reconversion}

As mentioned in section 2 mode conversionreconversion is a phenomenon existing in any over moded waveguide, such as would be used for longdistance transmission. The level of mode conversion or reconversion on their own is a function of waveguide tolerances, but the combined effect of mode conversion-recon version, which determines the signal distortion is also a function of waveguide design or more precisely it is related to the surface impedance of the waveguide.

For technological reasons one cannot reduce the mode conversion process beyond a certain limit, related to the waveguide tolerances. For given tolerances, however, the mode conversion-reconversion process can be reduced, in principle, below any predetermined level by choosing suitable surface impedance. ${ }^{5}$ The remaining problem then is a technological one, of realizing such surface impedance. The actual design is a compromise between what is desirable from the mode conversion-reconversion point of view and what is practicable or at all possible to achieve using existing methods.

Briefly, for good performance on bends, waveguides must have high surface reactance in the axial direction $\left(X_{z}\right.$ components) while the circumferential component $\left(X_{\phi}\right)$ remains small. For good discrimination against unwanted modes produced by typical small irregularities, large attenuation for unwanted modes and small attenuation for the $H_{01}$ mode is called for: that is large $R_{z}$ and small $R_{\phi}$.

Due to waveguide irregularities a large number of modes become coupled together. If the coupling coefficients representing the aggregate effect of waveguide irregularities contain a periodic component, considerable signal distortion takes place [4]: in effect the $\beta(\omega)$ characteristic becomes broken up by regularly spaced absorption bands. But if the irregularities are spaced in a random manner no such extreme signal distortion will take place; but on the other hand, the $\alpha$ and $\beta$ characteristics become irregular in a random manner with a root mean square value proportional to $(c / \Delta \beta)^{2}$ ( $c$ is the rms value of the coupling coefficient $c$, and $\Delta \beta$ the difference between the phase propagation coefficients of the $H_{01}$ mode and the parasitic mode) leading to effective crosstalk between the components of the coded signal. In general, the signal distortion due

${ }^{5}$ The conversion to $H_{o n}$ modes has been neglected, since this is not usually troublesome. to mode conversion-reconversion is a function of the quantity $(c / \Delta \beta)^{2} / \Delta \alpha$ where $\Delta \alpha$ is the difference between the attenuations of the parasitic mode and the $H_{01}$ mode. For this reason the more the group velocities of the parasitic modes differ from that of the $H_{01}$ mode and the greater the attenuation of the parasitic modes, the smaller the signal distortion due to mode conversion-reconversion. As discussed in section 4 , special waveguides can bring about such changes and hence their significance.

\section{Conclusions}

Waveguides for long distance communications already form a wide specialized subject, too wide to be discussed adequately in an article of this size. But we have discussed the main constituents of a waveguide communication system pointing out the necessity of regenerative modulation method, such as PCM.

Special waveguides have been discussed in some detail because, in the light of present knowledge, such waveguides - either of the helical type or dielectric coated - are the only types likely to succeed.

Mode conversion phenomena are intimately connected with overmoded waveguides, of the type used for long distance transmission. Deep understanding of mode conversion processes is essential for successful designs and accordingly a theory has been outlined. The design of such components as tapers, mode transducers, and bends rest on mode conversion theory.

Various sources of signal distortion have been analyzed in some detail, but it has been stressed that if PCM modulation is used and regenerative repeaters, as well as special waveguides, a most advanced system of unequalled capacity will result.

Most of the work described in this paper was carried out as part of a research program of Standard Telecommunication Laboratories Ltd. The authors have benefited from discussions with a number of their colleagues: such discussions helped to crystalize many of the more involved ideas.

Acknowledgment is also made to Standard Telecommunication Laboratories Ltd., for permission to publish the paper.

\section{References}

[1] S. E. Miller, Waveguide as a communication medium, Bell System Tech. J. 33, 1209 (1954).

[2] A. E. Karbowiak, Microwave aspects of waveguides for long distance transmission, Proc. Inst. Elec. Engrs. (London), Monograph No. 287R (Feb. 1958).

[3] H. M. Barlow, The exploration of waveguide for trunk waveguide multiple channel communications, J. Brit. Inst. Radio Engrs. 7, 251 (Oct. 1947).

[4] A. E. Karbowiak, Distortion of information in nonuniform multimode waveguides, Conv. on Long Distance Transmission by Waveguide, London (Jan. 1959). 
[5] A. E. Karbowiak and H. Knight, An experimental investigation of waveguides for long distance transmission, Conv. on Long Distance Transmission by Waveguide, London (Jan. 1959).

[6] A. E. Karbowiak, Propagation of transients in waveguides, Proc. Inst. Elec. Engrs. (London), Monograph No. 224R 105C (Feb. 1957).

[7] A. E. Karbowiak, Waveguide characteristics, Electron. and Radio Engr. 34, 379 (Oct. 1957).

[8] A. E. Karbowiak, Theory of imperfect guides-The effect of wall impedance, Proc. Inst. Elec. Engrs. (London) 102B, 698 (Sept. 1955).

[9] A. E. Karbowiak, Microwave propagation in anisotropic waveguides, Proc. Inst. Elec. Engrs. (London), Monograph No. 147R 103C, 139 (Aug. 1955).

[10] A. F. Stevenson, General theory of electromagnetic horns, J. Appl. Phys. 22, 1447 (Dec. 1951).

[11] S. A. Schelkunoff, Conversion of Maxwell's equations into generalised telegraphist's equations, Bell System Tech. J. 34, 995 (Sept. 1955).

[12] G. Reiter, Connection of two waveguides by a waveguide of variable cross-section (in Hungarian), Thesis from Appl. Math., Univ. Eotvos Lorand, Budapest (June 1955).

[13] G. Reiter, Generalised telegraphist's equation for waveguides of varying cross-section, Proc. Inst. Elec. Engrs. (London), pt. B Suppl. No. 13, p. 54 (Jan. 1959).

[14] B. Z. Katzenelenbaum, Non-uniform waveguides with slowly changing parameters (in Russian), Doklady Akad. Nauk. S.S.R. 12, 711 (1955).

[15] B. Z. Katzenelenbaum, On the general theory of nonuniform waveguides (in Russian), Dokl. Akad. Nauk. S.S.R. 116, 203 (1957).

[16] B. Z. Katzenelenbaum, On the theory of non-uniform waveguides with slowly changing parameters, Intern. Conf. Ultrahigh Frequency Circuits and Antennas, Paris (Oct. 1957).

[17] L. Solymar, Spurious mode generation in non-uniform waveguide, IRE Trans. MTT-y, 379 (July 1959).

[18] H. G. Unger, Circular waveguide taper of improved design, Bell System Tech. J. 37, 899 (July 1958).

[19] Y. Shimizu, Theory of transmitting circular electric wave around bends, presented at Intern. Conf. Ultrahigh Frequency Circuits and Antennas, Paris (Oct. 1957).

[20] M. Jouguet, Effect of curvature on the propagation of electromagnetic waves in guides of circular crosssection, Cables et Transmission 1, 133 (1947).
[21] W. J. Albersheim, Propagation of $\mathrm{TE}_{01}$ waves in curved waveguides, Bell System Tech. J. 28, 1 (1949).

[22] H. G. Unger, Normal mode bends for circular electric waves, Bell System Tech. J. 36, 1292 (1957).

[23] K. Tanaka, Mode conversion through the tapered section of circular waveguide system, Intern. Conf. Ultrahigh Frequency Circuits and Antennas, Paris (Oct. 1957).

[24] B. Z. Katzenelenbaum, Long symmetrical waveguide taper for $\mathrm{H}_{01}$ wave (in Russian), Radiotekh. Elektron. $\boldsymbol{2}^{1}, 531$ (1957).

[25] L. Solymar, Design of a conical taper in circular waveguide system supporting $\mathrm{H}_{01}$ mode, Proc. IRE 46, 618 (1958).

[26] L. Solymar, Design of a two-section conical taper in circular waveguide system supporting the $\mathrm{H}_{01}$ mode, Proc. Inst. Elec. Engrs. (London), pt. B. Suppl. No. 13, p. 119 (Jan. 1959).

[27] L. Solymar, Monotonic multi-section tapers for overmoded circular waveguides, Proc. Inst. Elec. Engrs. (London), pt. B. Suppl. No. 13, p. 121 (Jan. 1959).

[28] L. Solymar, Discussion on "Special microwave equipment," Proc. Inst. Elec. Engrs. (London), pt. B. Suppl. No. 13, p. 147 (Jan. 1959).

[29] L. Solymar and C. C. Eaglesfield, Design of mode transducers, IRE Trans. MTT-8, 61 (Jan. 1960).

[30] C. C. Eaglesfield, Y. Klinger, and L. Solymar, A new $\mathrm{H}_{10}$ to $\mathrm{H}_{20}$ mode transducer (to be published in Proc. Inst. Elec. Engrs. (London))

[31] B. Z. Katzenelenbaum, Imperfect waveguide in boundary and geometry, Izvest. Akad. Nauk., p. 9 (1955).

[32] S. P. Morgan, Mode conversion losses in transmission of circular electric waves through slightly non-circular guides, J. Appl. Phys. 21, 329 (1950).

[33] S. Iiguchi, Mode conversion in the transmission of $\mathrm{TE}_{01}$ wave through a slight tilt and a slight off-set of waveguide, Intern. Conf. Ultrahigh Frequency Circuits and Antennas, Paris (Oct. 1957).

[34] L. Solvmar, Overmoded waveguides, optical approach to mode conversion, Electron. and Radio Engr. 36, 426 (Nov. 1959).

[35] L. Solymar, Step transducer between overmoded circular waveguides, Proc. Inst. Elec. Engrs. (London), pt. B. Suppl. No. 13, p. 129 (Jan. 1959).

(Paper 65D1-105) 